\title{
Extending Chains of Factorizations and Minimal Negative Signatures
}

\author{
T. Constantinescu and $\mathbf{A}$. Gheondea
}

\begin{abstract}
We compute the minimal negative signature for a problem of extending chains of factorizations in spaces with indefinite inner product spaces. As an application, we obtain a formula for the minimal negative signature of Hermitian completions of tridiagonal partial block matrices.
\end{abstract}

Keywords: Indefinite factorization, negative signature, tridiagonal matrices

AMS subject classiflcation: 47B50, 47A20, 15A23

\section{Introduction}

A problem of extending factorizations of type ' $X^{t} X$ in Krein spaces was considered in [7] as a generalization of an abstract pattern of M. A. Naymark and M. G. Krein, in order to explore the intimate connection between the lifting of operators with control on the negative signature of defect, the lifting of commutants in Krein spaces and various interpolation problems.

The threshold of the above mentioned problems into the extending factorization problem is an abstract one-step completion of a partial block matrix operator. Motivated by other problems, there exists a parallel interest in problems of computing the negative signature, inertia, and rank for completions of partial matrices; as illustrated in $[4,5,8-12,15,17,18]$. The approach of these kind of problems has to face two main obstructions: the structure of the underlying graphs of the given partial matrices and the singularity of some submatrices combined with their interactions of certain kernels.

Given a partial Hermitian matrix $H$, i.e. a matrix in which certain entries are specified and the others are free, we denote by $\mathcal{C}_{h}(H)$ the set of all Hermitian completions $\tilde{H}$ of $H$. The problem we are interested in is to determine the number

$$
\min \left\{\kappa^{-}(\tilde{H}) \mid \tilde{H} \in \mathcal{C}_{h}(H)\right\} \text {. }
$$

Let us recall that $\kappa^{-}(H)$ denotes the negative signature if $H$ is a fully specified matrix and, in case $H$ is only a partial matrix, then it is defined as the maximum of the negative signatures of all its principal submatrices.

T. Constantinescu: Dept. of Math, Univ. of Texas at Dallas, Richardson, TX 75083-0688, USA; on leave from: Inst. de Mat., C.P. 1 - 764, RO - 70700 Bucureşti, România

A. Gheondea: Inst. de Mat., C.P. 1 - 764, RO - 70700 Bucureşti, România 
The problem whether there exist Hermitian completions $\tilde{H}$ such that $\kappa^{-}(\tilde{H})=$ $\kappa^{-}(H)$ was considered earlier by R. Grone, C. R. Johnson, E. M. de Sá, and H. Wolkowiecz [15] for the positive definite case (i.e. $\kappa^{-}(H)=0$ ), and by C. R. Johnnson and L. Rodman in [17] (for $\kappa^{-}(H)>0$ but with the additional requirement that some of the principal submatrices of $H$ are non-singular), when the role of the chordal graphs was also emphesized. In connection with this kind of problems the case of band matrices was intensively studied by H. Dym and I. Gohberg [10], R. E. Ellis, I. Gohberg and D. C. Lay [11], and I. Gohberg, M. A. Kaashoek and H. Woerdeman [13].

It was noticed just from the early beginning that in case the non-singularity assumption is dropped, it is possible that there exists no $\tilde{H} \in \mathcal{C}_{h}(H)$ such that $\kappa^{-}(\tilde{H})=\kappa^{-}(H)$ (see, e.g., [16]) and the calculation of the number in (1.1) becomes quite difficult. Different approaches for calculating this number have been used by J. Dancis $[8,9], J$. H. Maddocks [18], and the authors [5, 12].

The aim of this paper is to give a formula for computing the number in (1.1) for a tridiagonal block matrix, when no non-singularity is assumed. The technique we use is in the spirit of our approach in [7], that is, we formulate a problem of extending chains of factorizations of type $X^{b} X$. The main result for the abstract case is presented in Theorem 2.3. The novelty of this result, when compared with the corresponding result in [7], is that the tridiagonal case cannot be reduced to an iteration of the onestep completion, as in the positive definite case. The formula we obtain shows implicitly that this number reflects a global character of the chain of factorizations and emphasizes the interactions occuring between certain kernels.

We apply this result to the tridiagonal block operatorial matrix completions in Section 3. Then we specialize to the finite-dimensional case where the result (see Theorem 4.1 ) is in terms of the negative signatures of certain reduced Schur complements and certain numbers which "measure" the spatial position of the blocks with respect to the kernels of their intersection blocks. This enables us to obtain necessary and sufficient conditions for the existence of completions preserving the negative signature.

In this paper we use the same notation as in [7] and only occasionally recall some definitions, in order to avoid confusions. The small amount of facts from the theory of operators in Kreĭn spaces used here can be found in $[2,3]$, if not precisely indicated in the text.

\section{The problem of extending chains of factorizations}

Fix a positive integer $N$ and let there be given an $N$-tuple of Krein spaces $\left(\mathcal{K}_{i}\right)_{i=1}^{N}$ and an $(N+1)$-tuple of Krein spaces $\left(\mathcal{G}_{i}\right)_{i=1}^{N+1}$. Also, there are given operators $X_{i} \in \mathcal{L}\left(\mathcal{K}_{i}, \mathcal{G}_{i}\right)$ and $Y_{i} \in \mathcal{L}\left(\mathcal{K}_{i}, \mathcal{G}_{i+1}\right)$ such that

$$
\begin{aligned}
X_{i}^{\prime} X_{i} & =Y_{i}^{\prime} Y_{i}=Z_{i} \in \mathcal{L}\left(\mathcal{K}_{i}\right) & & (i \in\{1, \ldots, N\}) \\
X_{i+1}^{!} Y_{i} & =V_{i} \in \mathcal{L}\left(\mathcal{K}_{i}, \mathcal{K}_{i+1}\right) & & (i \in\{1, \ldots, N-1\})
\end{aligned}
$$

For simplicity we denote $\mathcal{X}=\left(X_{i}\right)_{i=1}^{N}$ and $\mathcal{Y}=\left(Y_{i}\right)_{i=1}^{N}$ and let $\underline{\kappa}=\left(\kappa_{i}\right)_{i=1}^{N+1}$ be an $(N+1)$-tuple of cardinal numbers. The problem of extending chains of factorizations has the following statement. 
Problem $\operatorname{EF}\left(\mathcal{X}, \mathcal{Y}_{i} \underline{\kappa}\right):$ Given $\mathcal{X}=\left(X_{i}\right)_{i=1}^{N}, \mathcal{Y}=\left(Y_{i}\right)_{i=1}^{N}$ and $\underline{\kappa}=\left(\kappa_{i}\right)_{i=1}^{N+1}$ such that (2.1) and (2.2) hold, it is required to determine (if any) a quadruple $(\underline{\mathcal{G}} ; \hat{\hat{\mathcal{X}}}, \hat{\mathcal{Y}} ; \mathcal{W})$ such that:

(a) $\underline{\mathcal{G}}=\left(\mathcal{G}_{i}^{\prime}\right)_{i=1}^{N+1}$, where $\mathcal{G}_{i}^{\prime}$ are Krein spaces with $\kappa^{-}\left[\mathcal{G}_{i}^{\prime}\right]=\kappa_{i}(i=1, \ldots, N)$.

(b) $\hat{\hat{X}}=\left(\hat{X}_{i}\right)_{i=1}^{N}$ and $\hat{\mathcal{Y}}=\left(\hat{Y}_{i}\right)_{i=1}^{N}$ are such that

(1) $\left.\hat{X}_{i} \in \mathcal{L}\left(\mathcal{K}_{i}, \mathcal{G}_{i} \mid+\right] \mathcal{G}_{i}^{\prime}\right)$ and $\hat{Y}_{i} \in \mathcal{L}\left(\mathcal{K}_{i}, \mathcal{G}_{i+1}[+] \mathcal{G}_{i+1}^{\prime}\right)$ are extensions of $X_{i}$ and $Y_{i}$, respectively $(i=1, \ldots, N)$.

(2) $\hat{X}_{i}^{t} \hat{X}_{i}=\hat{Y}_{i}^{\imath} \hat{Y}_{i}=Z_{i}(i=1, \ldots, N)$

(马) $\hat{X}_{i+1}^{i} \hat{Y}_{i}=V_{i}(i=1, \ldots, N-1)$.

(c) $W=\left(\hat{W}_{i}\right)_{i=1}^{N}$ are unitary operators such that

(1) $W_{i} \in \mathcal{L}\left(\mathcal{G}_{i+1}[+] \mathcal{G}_{i+1}^{\prime}, \mathcal{G}_{i}[+] \mathcal{G}_{i}^{\prime}\right)$, and $W_{i} \hat{Y}_{i}=\hat{X}_{i}(i=1, \ldots, N)$.

(2) $\bigvee_{i=0}^{N} W_{1} \cdots W_{i} \mathcal{G}_{i+1}=\mathcal{G}_{1}[+] \mathcal{G}_{1}^{\prime}$.

It is clear that letting $N=1$ we obtain the extending factorization problem as considered in [7]. Our main problem will be, similar as in [7], to determine the minimal $\underline{\kappa}$ such that problem $\operatorname{EF}(\mathcal{X}, \mathcal{Y} ; \underline{\kappa})$ is solvable. Before focusing on this we recall some facts and definitions.

We start with a first remark that we can assume, without restricting the generality, that all the Krein spaces $\mathcal{K}_{i}(i=1,2, \ldots, N)$ are positive definite (i.e. Hilbert spaces). Indeed, if this is not the case, then we fix on each Krein space $\mathcal{K}_{i}$ a fundamental symmetry $J_{i}$ and consider the induced Hilbert spaces $\left(\mathcal{K}_{i},(\cdot, \cdot) J_{i}\right)$. The corresponding problem $\operatorname{EF}(\mathcal{X}, \mathcal{Y} ; \underline{\kappa})$ has the same statement with the only difference that instead of $Z_{i}$ and $V_{i}$ we have $J_{i} Z_{i}$ and $J_{i} V_{i}$, respectively. From now on we assume that $\mathcal{K}_{i}$ are Hilbert spaces for all $i=1, \ldots, N$.

Let now $\mathcal{K}$ be a Krein space and $Z \in \mathcal{L}(\mathcal{K})$ be selfadjoint, $Z^{\prime}=Z$. We introduced in [6] the induced $K_{r}$ reln spaces $\mathcal{H}_{Z}$ and $\mathcal{K}_{Z}$ which are unitary equivalent (see the beginning of Section 3 for the definition of the Kreĭn space $\mathcal{K}_{Z}$ ). The Krein space $\mathcal{K}_{Z}$ is defined as follows: We fix a fundamental symmetry $J$ on $\mathcal{K}$, consider the polar decomposition of the selfadjoint operator (in a Hilbert space) $J Z$, let this be $J Z=S_{J Z}|J Z|$, and then define $\mathcal{H}_{Z}$ as the space $\mathcal{K} \ominus$ ker $J Z$, endowed with the new indefinite innner product defined by

$$
[x, y]=\left(S_{J Z} x, y\right)_{J} \quad \cdot(x, y \in \mathcal{K} \ominus \operatorname{ker} J Z) .
$$

Let $i \in\{1,2, \ldots, N\}$ be arbitrary. From Lemma 3.1 in [5] and (2.1), $X_{i}$ are uniquely represented by

$$
X_{i}=\left[V_{X_{i}}\left|Z_{i}\right|^{1 / 2} \quad X_{i}^{0}\right]
$$

where

$$
V_{X_{i}}: \mathcal{R}\left(\left|Z_{i}\right|^{1 / 2}\right)\left(\subseteq \mathcal{H}_{Z_{i}}\right) \longrightarrow \mathcal{G}_{i}
$$

is isometric such that $V_{X_{i}}\left|Z_{i}\right|^{1 / 2}$ is bounded and the operator

$$
X_{i}^{0}=X_{i} \mid \operatorname{ker} Z_{i} \in \mathcal{L}\left(\operatorname{ker} Z_{i}, \mathcal{G}_{i}\right)
$$

has its range $\mathcal{R}\left(X_{i}^{0}\right)$ neutral and included in $\mathcal{R}\left(V_{X_{i}}\right)^{\perp}$. In particular $\mathcal{R}\left(X_{i}^{0}\right)=\mathcal{R}\left(X_{i}\right)^{0}$ (the isotropic part of $\mathcal{R}\left(X_{i}\right)$ ). 
Following [7], the operator $X_{i}$ has the property $(\gamma)$ if:

( $\gamma$ ) The isometry $V_{X_{i}}$ in (2.9) extends (uniquely) in $\mathcal{L}\left(\mathcal{H}_{Z_{i}}, \mathcal{G}_{i}\right)$

¿From Lemma 3.2 in [7] this is equivalent with the condition that $X_{i} \mid\left(\operatorname{ker} Z_{i}\right)^{\perp}$ extends (uniquely) to an isometry in $\mathcal{L}\left(\mathcal{K}_{Z}, \mathcal{G}_{i}\right)$ and, as a consequence of this, $\overline{\mathcal{R}\left(X_{i}\right)}$ is a pseudoregular subspace of $\mathcal{G}_{i}$ and $\overline{\mathcal{R}\left(X_{i}\right)}=\overline{\mathcal{R}\left(X_{i}^{0}\right)}$.

Similar considerations can be made for the operators $Y_{i}$.

Coming back to the problem $\operatorname{EF}(\mathcal{X}, \mathcal{Y} ; \underline{\kappa})$, a special role will be played by the orthogonal projections in $\mathcal{K}_{i}$

$$
P_{i}=P_{X_{i}^{0 .}}^{\mathcal{K}_{i}} \quad \text { and } \quad Q_{i}=P_{Y_{i}^{0_{-}}}^{\mathcal{K}_{i}} \quad(i=1,2, \ldots, N)
$$

Also, recall that for two orthogonal projections $P$ and $Q$ acting in the Hilbert space $\mathcal{H}$, $P \wedge Q$ denotes the orthogonal projection of $\mathcal{H}$ onto $\mathcal{R}(P) \cap \mathcal{R}(Q)$.

Proposition 2.1. Assume that for all $i \in\{1,2, \ldots, N\}$ the operators $X_{i}$ and $Y_{i}$ have the property $(\gamma)$ and the projections $P_{i} \wedge Q_{i}$ have finite ranks. If the problem $\operatorname{EF}(\mathcal{X}, \mathcal{Y} ; \underline{\kappa})$ has solutions, then

$$
\begin{aligned}
& \kappa_{i}+\operatorname{rank}\left(P_{i}\left(I-Q_{i}\right)\right)+\kappa^{-}\left[\mathcal{R}\left(X_{i}\right)^{\perp}\right] \\
& \quad=\kappa_{i+1}+\operatorname{rank}\left(Q_{i}\left(I-P_{i}\right)\right)+\kappa_{-}\left[\mathcal{R}\left(Y_{i}\right)^{\perp}\right] \quad(i=1, \ldots, N)
\end{aligned}
$$

and

$$
\begin{aligned}
\kappa_{1} & \geq \operatorname{rank}\left(Q_{1}\left(I-P_{1}\right)\right) \\
\kappa_{i} & \geq \max \left\{\operatorname{rank}\left(P_{i-1}\left(I-Q_{i-1}\right)\right), \operatorname{rank}\left(Q_{i}\left(I-P_{i}\right)\right)\right\} \quad(i=1, \ldots, N) \\
\kappa_{N+1} & \geq \operatorname{rank}\left(P_{N}\left(I-Q_{N}\right)\right)
\end{aligned}
$$

\section{must hold.}

Proof. From Lemma 3.3 in [7] we know that for all $i \in\{1,2, \ldots, N\}$ we have

$$
\kappa_{i}+\operatorname{rank}\left(P_{i}\right)+\kappa^{-}\left[\mathcal{R}\left(X_{i}\right)^{\perp}\right]=\kappa_{i+1}+\operatorname{rank}\left(Q_{i}\right)+\kappa^{-\left[\mathcal{R}\left(Y_{i}\right)^{\perp}\right]}
$$

On the other hand, taking into account the decompositions

$$
\begin{aligned}
& \mathcal{R}\left(P_{i}\right)=\mathcal{R}\left(P_{i} \wedge Q_{i}\right) \oplus \mathcal{R}\left(P_{i}\left(I-Q_{i}\right)\right) \\
& \mathcal{R}\left(Q_{i}\right)=\mathcal{R}\left(P_{i} \wedge Q_{i}\right) \oplus \mathcal{R}\left(Q_{i}\left(I-P_{i}\right)\right)
\end{aligned}
$$

we subtract rank $\left(P_{i} \wedge Q_{i}\right)$ from both sides in (2.7) and obtain the equation (2:5). The inequalities (2.6) are obtained exactly as in the proof of Theorem 3.4 of [7] 
The linear relations (2.5) and the constraints (2.6) are the basic conditions that $\underline{\kappa}=\left(\kappa_{i}\right)_{i=1}^{N+1}$ satisfy. If some of the quantities appearing there are infinite, then there are many possibilities for $\underline{\kappa}$ such that problem $\operatorname{EF}(\mathcal{X}, \mathcal{Y} ; \underline{\kappa})$ has solutions. However, if all these quantities are finite, then the set of $(N+1)$-tuples $\underline{\kappa}$ for which problem $\operatorname{EF}(\mathcal{X}, \mathcal{Y} ; \underline{\kappa})$ has solutions depends only on one linear parameter, which can be chosen one of $\kappa_{i}$. In particular, in this case, the problem of determining the minimal value of $\underline{\kappa}$ for which the problem $\operatorname{EF}(\mathcal{X}, \mathcal{Y} ; \underline{\kappa})$ has solutions does not depend on which order relation on $\mathbb{R}^{N+1}$ (e.g. product order, lexicographic order) we consider.

For clarity reasons we consider first a linear programming problem which yields the minimal value of $\underline{\kappa}$ in a certain case. Let $\left\{a_{i 1}, a_{i 2}\right\}_{i=1}^{N}$ and $\left\{r_{i-1,2}, r_{i 1}\right\}_{i=1}^{N+1}$ consists of real scalars and let us consider the relations

$$
\begin{array}{rlrl}
\kappa_{i}+r_{i 2}+a_{i 1} & =\kappa_{i+1}+r_{i 1}+a_{i 2} & & (1 \leq i \leq N) \\
\kappa_{i} \geq \max \left\{r_{i-1,2}, r_{i 1}\right\} & & (1 \leq i \leq N+1)
\end{array}
$$

for an $(N+1)$-tuple $\underline{\kappa}=\left(\kappa_{i}\right)_{i=1}^{N+1}$.

Lemma 2.2. Let $j \in\{1,2, \ldots, N+1\}$ be determined such that

$$
\begin{aligned}
& \max \left\{r_{j-1,2}, r_{j 1}\right\}+\sum_{k=1}^{j-1}\left[\left(a_{k 2}-a_{k 1}\right)+\left(r_{k 1}-r_{k 2}\right)\right] \\
& =\max _{n=1}^{N+1}\left\{\max \left\{r_{n-1,2}, r_{n 1}\right\}+\sum_{k=1}^{n-1}\left[\left(a_{k 2}-a_{k 1}\right)+\left(r_{k 1}-r_{k 2}\right)\right]\right\}
\end{aligned}
$$

and then define $\underline{\kappa}^{\min }=\left(\kappa_{i}^{\min }\right)_{i=1}^{N+1}$ as

$$
\begin{aligned}
& \kappa_{i}^{\min }= \\
& \quad \begin{cases}\max \left\{r_{j-1,2}, r_{j 1}\right\}+\sum_{k=i}^{j-1}\left[\left(a_{k 2}-a_{k 1}\right)+\left(r_{k 1}-r_{k 2}\right)\right] & \text { if } 1 \leq i<j \\
\max \left\{r_{j-1,2}, r_{j 1}\right\} & \text { if } i=j \\
\max \left\{r_{j-1,2}, r_{j 1}\right\}+\sum_{k=j}^{i-1}\left[\left(a_{k 2}-a_{k 1}\right)+\left(r_{k 1}-r_{k 2}\right)\right] & \text { if } j<i \leq N+1\end{cases}
\end{aligned}
$$

Then $\underline{\kappa}^{\min }$ is the minimal $(N+1)$-tuple satisfying the linear system (2.5) and the linear constraints (2.9).

Proof. We first note that in (2.11) we use the convention that a sum whose beginning index of summation is greater than the ending index of summation is null.

The linear system (2.5) represents a straight line in $\mathbb{R}^{N+1}$ which is equally inclined with respect to each of the $N+1$ coordinate axis. On the other hand, the constraints (2.9) represents a rectangle whose $N+1$ faces are parallel, respectively, with respect to the $N+1$ hyperplanes determined by the coordinate axis. Thus, there exists and it is unique the minimum of $\underline{\kappa}$ satisfying the conditions (2.5) and (2.9). Moreover, denoting by $\underline{\kappa}^{\text {min }}$ this minimal solution, there exists at least one index $j \in\{1,2, \ldots, N+1\}$ such that $\kappa_{j}^{\min }=\max \left\{r_{j-1,2}, r_{j 1}\right\}$. The fact that this index $j$ can be chosen as in (2.10) is a simple verification and now the formulae (2.11) are produced from (2.5) 
Coming back to the problem $\operatorname{EF}(\mathcal{X}, \mathcal{Y} ; \underline{\kappa})$ we make the following notation:

$$
\begin{aligned}
r_{i 1}=\operatorname{rank}\left(Q_{i}\left(I-P_{i}\right)\right), & r_{i 2}=\operatorname{rank}\left(P_{i}\left(I-Q_{i}\right)\right) \\
a_{i 1}=\kappa^{-}\left[\mathcal{R}\left(X_{i}\right)^{\perp}\right], & a_{i 2}=\kappa^{-}\left[\mathcal{R}\left(Y_{i}\right)^{\perp}\right]
\end{aligned}
$$

for all $i \in\{1,2, \ldots, N\}$ and, by definition, we put $r_{01}=r_{N+1,2}=0$.

We are now in a position to prove our main result.

Theorem 2.3. Assume that the operators $X_{i}$ and $Y_{i}$ have the property $(\gamma)$ and also that the numbers $\operatorname{rank}\left(P_{i}\right), \operatorname{rank}\left(Q_{i}\right)$ and $\kappa^{-}\left[\mathcal{R}\left(X_{i}\right)^{\perp}\right], \kappa^{-}\left[\mathcal{R}\left(Y_{i}\right)^{\perp}\right]$ are all finite. Then, with the notation stated in (2.12) and (2.13), the $(N+1)$-tuple $\kappa^{\min }$ defined in Lemma 2.2 is the minimal value of the $(N+1)$-tuples $\underline{\kappa}$ for which the problem $\operatorname{EF}(\mathcal{X}, \mathcal{Y} ; \underline{\kappa})$ is solvable.

Proof. From the Proposition 2.1 and Lemma 2.2 it follows that if $\underline{\kappa}$ is an $(N+1)$ tuple for which the problem $\operatorname{EF}(\mathcal{X}, \mathcal{Y} ; \underline{\kappa})$ is solvable, then $\underline{\kappa} \geq \underline{\kappa}^{\min }$, where $\underline{\kappa}^{\min }$ is defined in Lemma 2.2.

Conversely, we will show that problem $\operatorname{EF}\left(\mathcal{X}, \mathcal{Y} ; \underline{\kappa}^{\min }\right)$ is solvable by producing an explicit solution. To this end, let us first notice that, for an arbitrary $i \in\{1,2, \ldots, N\}$, since $X_{i}$ and $Y_{i}$ have the property $(\gamma)$, we have the decompositions

$$
\begin{aligned}
\mathcal{G}_{i} & =\mathcal{L}_{i}[+]\left[\mathcal{R}\left(X_{i}^{0}\right) \oplus J_{i} \mathcal{R}\left(X_{i}^{0}\right)\right][+] \mathcal{L}_{i}^{\prime} \\
\mathcal{G}_{i+1} & =\mathcal{S}_{i}[+]\left[\mathcal{R}\left(Y_{i}^{0}\right) \oplus J_{i+1} \mathcal{R}\left(Y_{i}^{0}\right)\right][+] \mathcal{S}_{i}^{\prime}
\end{aligned}
$$

where $\mathcal{L}_{i}, \mathcal{L}_{i}^{\prime}, \mathcal{S}_{i}$ and $\mathcal{S}_{i}^{\prime}$ are regular subspaces such that

$$
\mathcal{R}\left(X_{i}\right)^{\perp}=\mathcal{R}\left(X_{i}^{0}\right)[+] \mathcal{L}_{i}^{\prime} \quad \text { and } \quad \mathcal{R}\left(Y_{i}\right)^{\perp}=\mathcal{R}\left(Y_{i}^{0}\right)[+] \mathcal{S}_{i}^{\prime}
$$

and $J_{i}$ are (fixed) fundamental symmetries on each of the Krein spaces $\mathcal{G}_{i}$.

If $\mathcal{H}$ is a Hilbert space, then we denote by $[\mathcal{H} \oplus \mathcal{H}]$ the Krein space obtained from the Hilbert space $\mathcal{H} \oplus \mathcal{H}$ with the fundamental symmetry $J$ defined by $J=\left(\begin{array}{ll}0 & I \\ I & 0\end{array}\right)$. We consider now a family of Krein spaces $\left\{\mathcal{G}_{i 1}\right\}_{i=1}^{N+1}$, subject to the following conditions:

(1) $\mathcal{G}_{i 1}$ contains $\left[\mathcal{H}_{i 1} \oplus \mathcal{H}_{i 1}\right]$ as a regular subspace, where $\mathcal{H}_{i 1}$ is a copy of the Hilbert space $\mathcal{R}\left(Q_{i}\left(I-P_{i}\right)\right)$.

(2) $\mathcal{G}_{i+1,1}$ contains $\left[\mathcal{H}_{i 2} \oplus \mathcal{H}_{i 2}\right]$ as a regular subspace, where $\mathcal{H}_{i 2}$ is a copy of the Hilbert space $\mathcal{R}\left(P_{i}\left(I-Q_{i}\right)\right)$.

(3) If $r_{i-1,2} \geq r_{i 1}$, then $\mathcal{H}_{i 1} \subseteq \mathcal{H}_{i-1,2}$ and if $r_{i-1,2} \leq r_{i 1}$, then $\mathcal{H}_{i-1,2} \subseteq \mathcal{H}_{i 1}$.

(4) $\kappa^{-}\left[\mathcal{G}_{i 1}\right]=\kappa_{i}^{\mathrm{min}}$.

Then, let $X_{i}^{1}$ be the embedding on the first component

$$
X_{i}^{1}: \mathcal{R}\left(Q_{i}\left(I-P_{i}\right)\right) \hookrightarrow\left[\mathcal{H}_{i 1} \oplus \mathcal{H}_{i 1}\right]
$$

and similarly, let $Y_{i}^{1}$ be the embedding on the first component

$$
Y_{i}^{1}: \mathcal{R}\left(P_{i}\left(I-Q_{i}\right)\right) \hookrightarrow\left[\mathcal{H}_{i 2} \oplus \mathcal{H}_{i 2}\right]
$$


and then extend $X_{i}^{1}$ and $Y_{i}^{1}$ trivially onto the whole ker $Z_{i}$. Using these we define

$$
\hat{X}_{i}^{0}=\left[\begin{array}{ll}
X_{i}^{0} & X_{i}^{1}
\end{array}\right]^{t}: \operatorname{ker} Z_{i} \rightarrow \mathcal{G}_{i}[+]\left[\mathcal{H}_{i 1} \oplus \mathcal{H}_{i 1}\right]
$$

and similarly

$$
\hat{Y}_{i}^{0}=\left[\begin{array}{ll}
Y_{i}^{0} & Y_{i}^{1}
\end{array}\right]^{t}: \operatorname{ker} Z_{i} \rightarrow \mathcal{G}_{i+1}[+]\left[\mathcal{K}_{i 2} \oplus \mathcal{H}_{i 2}\right] .
$$

With these definitions it is easy to check that $\mathcal{R}\left(\hat{X}_{i}^{0 *}\right)=\mathcal{R}\left(\hat{Y}_{i}^{0 *}\right)$, consequently, there exists an invertible operator $T_{i}: \mathcal{R}\left(\hat{Y}_{i}^{0}\right) \rightarrow \mathcal{R}\left(\hat{X}_{i}^{0}\right)$ such that $T_{i} \hat{Y}_{i}^{0}=\hat{X}_{i}^{0}$ which (e.g. by Lemma 2.4 in [7]) can be extended to a unitary operator

$$
\begin{aligned}
& W_{i}:\left(\mathcal{R}\left(Y_{i}^{0}\right) \oplus J_{i+1} \mathcal{R}\left(Y_{i}^{0}\right)\right)[+]\left[\mathcal{H}_{i 2} \oplus \mathcal{H}_{i 2}\right] \\
& \quad \rightarrow\left(\mathcal{R}\left(X_{i}^{0}\right) \oplus J_{i} \mathcal{R}\left(X_{i}^{0}\right)\right)[+]\left[\mathcal{H}_{i 1} \oplus \mathcal{H}_{i 1}\right]
\end{aligned}
$$

such that

$$
W_{i} \hat{Y}_{i}^{0}=\hat{X}_{i}^{0}
$$

Define

$$
\begin{aligned}
\hat{X}_{i} & =\left[\begin{array}{ll}
V_{X_{i}}\left|Z_{i}\right|^{1 / 2} & \hat{X}_{i}^{0}
\end{array}\right] \\
\hat{Y}_{i} & =\left[\begin{array}{ll}
V_{Y_{i}}\left|Z_{i}\right|^{1 / 2} & \hat{Y}_{i}^{0}
\end{array}\right]
\end{aligned}
$$

and then extend $W_{i}$ with $V_{X_{i}} V_{Y_{i}}^{-1}$. In particular, we have

$$
W_{i} \hat{Y}_{i}=\hat{X}_{i}
$$

On the other hand, since $\underline{\kappa}^{\min }$ is a solution of the system (2.5) it follows that there exists a family of doubly contractive operators $\left\{C_{i}\right\}_{i=1}^{N}$ such that

$$
C_{i}: \mathcal{L}_{i}^{\prime}[+]\left(\mathcal{G}_{i+1,1} \cap\left[\mathcal{H}_{i 2} \oplus \mathcal{H}_{i 2}\right]^{\perp}\right) \rightarrow \mathcal{S}_{i}^{\prime}[+]\left(\mathcal{G}_{i, 1} \cap\left[\mathcal{H}_{i 1} \oplus \mathcal{H}_{i 1}\right]^{\perp}\right) .
$$

Let us denote by

$$
\mathcal{G}_{i 2}^{\prime}=\mathcal{L}_{i}^{\prime}[+]\left(\mathcal{G}_{i+1,1} \cap\left[\mathcal{H}_{i 2} \oplus \mathcal{H}_{i 2}\right]^{\perp}\right)
$$

the domain of $C_{i}$, and also denote by

$$
\mathcal{G}_{i 2}^{\prime \prime}=\mathcal{S}_{i}^{\prime}[+]\left(\mathcal{G}_{i, 1} \cap\left[\mathcal{H}_{i 1} \oplus \mathcal{H}_{i 1}\right]^{\perp}\right)
$$

its range space. We introduce now two families of Krein spaces $\left\{\mathcal{F}_{i}^{\prime}\right\}_{i=1}^{N}$ and $\left\{\mathcal{F}_{i}^{\prime}\right\}_{i=1}^{N}$ as follows:

$$
\begin{aligned}
\mathcal{F}_{i}^{\prime \prime} & =\mathcal{D}_{C_{i}}[+] \mathcal{D}_{C_{i}^{*}[+]} \cdots[+] \mathcal{D}_{C_{i-1}^{*}}[+] \mathcal{G}_{i 2}^{\prime \prime}[+] \mathcal{D}_{C_{i}}[+] \cdots[+] \mathcal{D}_{C_{N}} \\
\mathcal{F}_{i}^{\prime} & =\mathcal{D}_{C_{i}}[+] \mathcal{D}_{C_{i}^{*}}[+] \cdots[+] \mathcal{D}_{C_{i}^{*}}[+] \mathcal{G}_{i 2}^{\prime}[+] \mathcal{D}_{C_{i+1}}[+] \cdots[+] \mathcal{D}_{C_{N}}
\end{aligned}
$$

and using these we define the unitary operators $W_{i}^{\prime} \in \mathcal{L}\left(\mathcal{F}_{i}^{\prime}, \mathcal{F}_{i}^{\prime \prime}\right)$ by

$$
\left.W_{i}^{\prime}=I_{i-1,1} \mid+\right] R\left(C_{i}\right)|+| I_{N-1,2}
$$


where $R\left(C_{i}\right) \in \mathcal{L}\left(\mathcal{G}_{i 2}^{\prime}[+] \mathcal{D}_{C_{i}^{*}}, \mathcal{G}_{i 2}^{\prime \prime}[+] \mathcal{D}_{C_{i}}\right)$ denotes the elementary rotation of $C_{i}$ (see [1] for the definition and the proof of the existence), $I_{i-1,1}$ denotes the identity operator on the Kreĭn space $\mathcal{D}_{C_{1}^{*}}\left[+\mid \mathcal{D}_{C_{2}^{*}}[+] \cdots[+] \mathcal{D}_{C_{i-1}^{*}}\right.$ and $I_{N-1,2}$ denotes the identity operator on the Kreĭn space $\mathcal{D}_{C_{i+1}}[+] \cdots[+] \mathcal{D}_{C_{N}}$.

Finally, let us define the family of Krein spaces $\left\{\mathcal{G}_{i}^{\prime}\right\}_{i=1}^{N+1}$ by

$$
\left.\mathcal{G}_{i}^{\prime}=\mathcal{G}_{i 1}[+] \mathcal{D}_{C_{i}}[+] \mathcal{D}_{C_{i}}[+] \cdots[+] \mathcal{D}_{C_{i}}[+] \mathcal{D}_{C_{i}} l+\right] \cdots[+] \mathcal{D}_{C_{N}}
$$

and then extend $\left.\left.W_{i} \in \mathcal{L}\left(\mathcal{G}_{i+1} \mid+\right] \mathcal{G}_{i+1}^{\prime}, \mathcal{G}_{i} \mid+\right] \mathcal{G}_{i}^{\prime}\right)$ with the unitary operator $W_{i}^{\prime}$. Extending trivially $\hat{X}_{i}$ and $\hat{Y}_{i}$ such that

$$
\mathcal{R}\left(\hat{X}_{i}\right) \subseteq \mathcal{G}_{i}[+] \mathcal{G}_{i}^{\prime} \quad \text { and } \quad \mathcal{R}\left(\hat{Y}_{i}\right) \subseteq \mathcal{G}_{i+1}[+] \mathcal{G}_{i+1}^{\prime}
$$

we claim that this way we have obtained a solution of the problem $\operatorname{EF}\left(\mathcal{X}, \mathcal{Y} ; \underline{\kappa}^{\mathrm{min}}\right)$.

Indeed, condition (a) holds since all of $C_{i}$ are doubly contractive, i.e. $\mathcal{D}_{C_{i}}$ and $\mathcal{D}_{C_{i}}$ are positive definite and hence, from (2.17) and the assumption (3) on $\mathcal{G}_{i 1}$, we have

$$
\kappa^{-}\left[\mathcal{G}_{i}^{\prime}\right]=\kappa^{-}\left[\mathcal{G}_{i 1}\right]=\kappa_{i}^{\min } .
$$

The condition (b) follows from the construction of $\hat{X}_{i}$ and $\hat{Y}_{i}$ and taking into account of

$$
\mathcal{R}\left(\hat{X}_{i}^{0}\right) \perp \mathcal{R}\left(\hat{Y}_{i-1}^{0}\right)
$$

The condition (c)/(1) follows since the extensions of $\hat{X}_{i}$ and $\hat{Y}_{i}$ after proving (2.16) do not alter their ranges and, finally, the condition $(c) /(2)$ follows from the minimality property of the elementary rotation (see [6])

\section{Completions of tridiagonal block matrix operators}

Before stating the problem to be investigated in this section we need first to recall some definitions and facts concerning selfadjoint two-by-two operators, in connection with induced Kreĭn spaces.

First recall that given a selfadjoint operator $A \in \mathcal{L}(\mathcal{H})$, one defines a Krein space $\left(\mathcal{K}_{A},[\cdot, \cdot]_{A}\right)$ as being the completion of $\mathcal{H} \ominus \operatorname{ker} A$ under a certain Hilbert norm, the indefinite inner product $\left[\cdot, \cdot \dot{j}_{A}\right.$ being defined as $[x, y]_{A}=(A x, y)(x, y \in \mathcal{H} \ominus \operatorname{ker} A)$. In addition, there exists the canonical mapping $\pi_{A} \in \mathcal{R}\left(\mathcal{H}, \mathcal{K}_{A}\right)$, by definition this is the composition of the quotient mapping with the natural inclusion, and it holds

$$
\pi_{A}^{\prime} \pi_{A}=A
$$

Let now $\mathcal{H}_{1}$ and $\mathcal{H}_{2}$ be Hilbert spaces and let also $H \in \mathcal{L}\left(\mathcal{H}_{1} \oplus \mathcal{H}_{2}\right)$ be a two-by-two matrix operator

$$
H=\left[\begin{array}{cc}
A & B \\
B^{*} & C
\end{array}\right]
$$


Consider the operator $\rho_{H, C} \in \mathcal{L}\left(\mathcal{H}_{2}, \mathcal{K}_{H}\right)$ defined by

$$
\rho_{H, C} h=\pi_{H} h \quad\left(h \in \mathcal{H}_{2}\right) .
$$

This yields

$$
\rho_{H, C}^{\prime} \rho_{H, C}=C,
$$

hence the linear operator $\rho_{H, C} \mid \mathcal{H}_{2} \ominus \operatorname{ker} C: \mathcal{H}_{2} \ominus \operatorname{ker} C\left(\subseteq \mathcal{K}_{C}\right) \rightarrow \mathcal{K}_{H}$ is isometric. By definition, the Krein space $\mathcal{K}_{C}$ is canonically embedded in $\mathcal{K}_{H}$ if this isometric operator is bounded and, in this case, the Krein space $\mathcal{K}_{C}$ is identified with the regular subspace $\rho_{H, C} \mathcal{K}_{C}$ of $\mathcal{K}_{H}$. It is easy to see that this is equivalent with the condition that, with respect to the factorization (3.1), the operator $\rho_{H, C}$ have the property $(\gamma)$.

There are several criteria assuring that $\mathcal{K}_{C}$ be canonically embedded in $\mathcal{K}_{H}$. Here we recall the one considered in [7: Lemma 4.3].

Lemma 3.1. Assume that the operator $B_{1}=B \mid \mathcal{H}_{2} \ominus \operatorname{ker} C$ is factored by the operator $C$ and denote $S_{\mathrm{r}}(H ; C)=P_{\text {ker } B_{i}}\left(A-B_{1} C^{-1} B_{1}^{*}\right) \mid \operatorname{ker} B_{2}^{*}$ where $B_{2}=B \mid \operatorname{ker} C$ and the inverse of $C$ is defined on its range. Then $\mathcal{K}_{C}$ is canonically embedded into $\mathcal{K}_{H}$ and, in addition, $\kappa^{ \pm}\left[\mathcal{R}\left(\rho_{H, C}\right)^{\perp}\right]=\kappa^{ \pm}\left(S_{r}(H ; C)\right)$.

The operator $S_{r}(H ; C)$ defined in Lemma 3.1 (if it exists) is called the reduced Schur complement of $H$ with respect to $C$.

Let us also record that $\mathcal{R}\left(\rho_{H, C} \mid \operatorname{ker} C\right)$ is the isotropic part of $\mathcal{R}\left(\rho_{H, C}\right)$ and, as a consequence of [6: Lemma 4.2], we have

$$
\operatorname{rank}\left(\rho_{H, C} \mid \operatorname{ker} C\right)=\operatorname{rank}(B \mid \operatorname{ker} C)
$$

Also, given a Kreìn space $\mathcal{G}$, we say that the decomposition $\mathcal{K}_{H}=\mathcal{K}_{C}[+\mid \mathcal{G}$ holds modulo canonical embedding, if there exists a unitary operator $U: \mathcal{K}_{C}[+] \mathcal{G} \rightarrow \mathcal{K}_{H}$ such that $U \mid \mathcal{K}_{C}$ coincides with the canonical embedding of $\mathcal{K}_{C}$ into $\mathcal{K}_{H}$.

In order to state the completion problem we consider a symmetric tridiagonal partial block matrix operator, let this be

$$
H=\left[\begin{array}{cccccc}
A_{11} & A_{12} & & & & \\
A_{12}^{*} & A_{22} & A_{23} & & & \\
\ddots & A_{23}^{*} & A_{33} & \ddots & \ddots & \\
& & \ddots & \ddots & \ddots & \\
& & & \ddots & A_{N+1, N+1} & A_{N+1, N+2} \\
& & & & A_{N+1, N+2}^{*} & A_{N+2, N+2}^{*}
\end{array}\right] .
$$

Here the operator $H$ is supposed to "act", on the Hilbert space

$$
\mathcal{H}=\mathcal{H}_{1} \oplus \mathcal{H}_{2} \oplus \cdots \oplus \mathcal{H}_{N+2}
$$

or, equivalently, $A_{i i} \in \mathcal{L}\left(\mathcal{H}_{i}\right)$ for $i \in\{1, \ldots, N+2\}$ and $A_{i, i+1} \in \mathcal{L}\left(\mathcal{H}_{i+1}, \mathcal{H}_{i}\right)$ for $i \in$ $\{1, \ldots, N+1\}$. In order to hold the symmetry of $H$ we assume that $A_{i i}$ are selfadjoint. 
By definition, a selfadjoint completion of $H$ is a selfadjoint operator $\tilde{H} \in \mathcal{L}(\mathcal{H})$ such that, for all $i \in\{1, \ldots, N+1\}$,

$$
P_{\mathcal{H}_{i} \oplus \mathcal{H}_{i+1}} \tilde{H}\left|\mathcal{H}_{i} \oplus \mathcal{H}_{i+1}=P_{\mathcal{H}_{i} \oplus \mathcal{H}_{i+1}} H\right| \mathcal{H}_{i} \oplus \mathcal{H}_{i+1}
$$

It is helpful to introduce the operators $H_{i} \in \mathcal{L}\left(\mathcal{H}_{i} \oplus \mathcal{H}_{i+1}\right)$

$$
H_{i}=\left[\begin{array}{cc}
A_{i i} & A_{i, i+1} \\
A_{i, i+1}^{*} & A_{i+1, i+1}
\end{array}\right]
$$

The selfadjoint operators $H_{i}$ are viewed as maximal fully specified submatrices of $H$. In this context we can figure the partial matrix of $H$ using the fully specified $\dot{H}_{i}$ as follows:

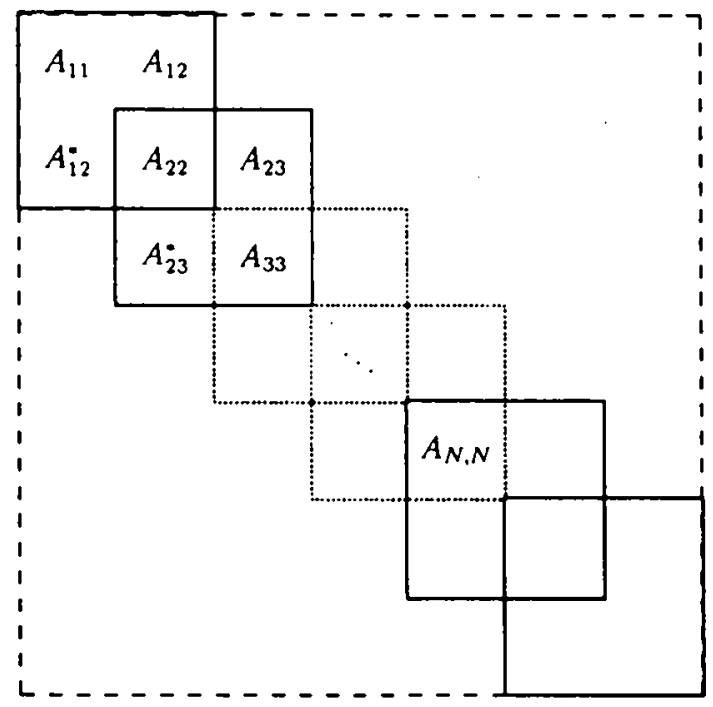

Figure 1

We are now in a position to formulate the completion problem for chains of factorizations.

Problem $\mathbf{C}(H ; \underline{\kappa})$ : Given a symmetric tridiagonal partial block matrix operator $H$ as in (9.6) and an $(N+1)$-tuple $\underline{\kappa}=\left(\kappa_{i}\right)_{i=1}^{N+1}$ of cardinal numbers, it is required to determine a pair $\left(\tilde{H}_{;}\left(\mathcal{G}_{i}^{\prime}\right)_{i=1}^{N+1}\right)$ such that

(a) $\mathcal{G}_{i}^{\prime}$ are $K$ rein spaces and $\kappa^{-}\left[\mathcal{G}_{i}^{\prime}\right]=\kappa_{i}$ for all $i \in\{1,2, \ldots, N+1\}$

(b) $\tilde{H} \in \mathcal{L}(\mathcal{H})$ is a selfadjoint completion of $H$ such that, modulo canonical embeddings, for all $i \in\{1, \ldots, N+1\}$ we have $\mathcal{K}_{\dot{H}}=\mathcal{K}_{H_{i}}\left[+\mid \mathcal{G}_{i}^{\prime}\right.$.

We will first show that the problem $\mathbf{C}(H ; \underline{\kappa})$ can be put into the framework of a problem of type $\operatorname{EF}\left(\mathcal{X}, \mathcal{Y} ; \underline{\kappa}^{\text {min }}\right)$. To this end, let us assume that the problem $\mathbf{C}(H ; \underline{\kappa})$ 
has at least one solution $\left(\tilde{H}_{;}\left(\mathcal{G}_{i}^{\prime}\right)_{i=1}^{N+1}\right)$. We consider the families of operators $\mathcal{X}=$ $\left(X_{i}\right)_{i=1}^{N}$ and $\mathcal{Y}=\left(Y_{i}\right)_{i=1}^{N}$ defined by

$$
X_{i}=\pi_{H_{i}} \mid \mathcal{H}_{i+1} \quad \text { and } \quad Y_{i}=\pi_{H_{i+1}} \mid \mathcal{H}_{i+1} .
$$

We take $\mathcal{K}_{i}=\mathcal{H}_{i+1}$, for all $i \in\{1, \ldots, N\}$. Using (3.1) it is now easy to see that

$$
X_{i}^{l} X_{i}=Y_{i}^{l} Y_{i}=A_{i+1, i+1}^{*} \quad \text { and } \quad X_{i+1}^{i} Y_{i}=A_{i+1, i+2}
$$

for all possible $i$, hence we can formulate the problem $\operatorname{EF}(\mathcal{X}, \mathcal{Y} ; \underline{\kappa})$, with the same $\underline{\kappa}$ as in the problem $\mathrm{C}(H ; \underline{\kappa})$ and, in addition, $Z_{i}=A_{i+1, i+1}^{*}$ and $V_{i}=A_{i+1, i+2}$. By definition, there exist unitary operators $\omega_{i}: \mathcal{K}_{\mathcal{H}_{i}}|+| \mathcal{G}_{i}^{\prime} \rightarrow \mathcal{K}_{\bar{H}}$, for all $i \in\{1, \ldots, N+1\}$. Using these we consider the family of operators $\mathcal{W}=\left(W_{i}\right)_{i=1}^{N}$ defined by

$$
W_{i}=\omega_{i}^{-1} \omega_{i+1}
$$

and then define the families of operators $\hat{\mathcal{X}}=\left(\hat{X}_{i}\right)_{i=1}^{N}$ and $\hat{\mathcal{Y}}=\left(\hat{Y}_{i}\right)_{i=1}^{N}$ by

$$
\hat{X}_{i}=\omega_{i}^{-1} \pi_{\dot{H}} \mid \mathcal{K}_{i} \quad \text { and } \quad \hat{Y}_{i}=\omega_{i+1}^{-1} \pi_{\dot{H}} \mid \mathcal{K}_{i} .
$$

It is useful to consider all the previous constructions through a diagram (see Figure 2) which is drawn for all possible $i$. The symbol "///", placed in the centre of a triangular diagram, means that the corresponding diagram is commutative. The vertical or horizontal arrows having no name are supposed to be embeddings.

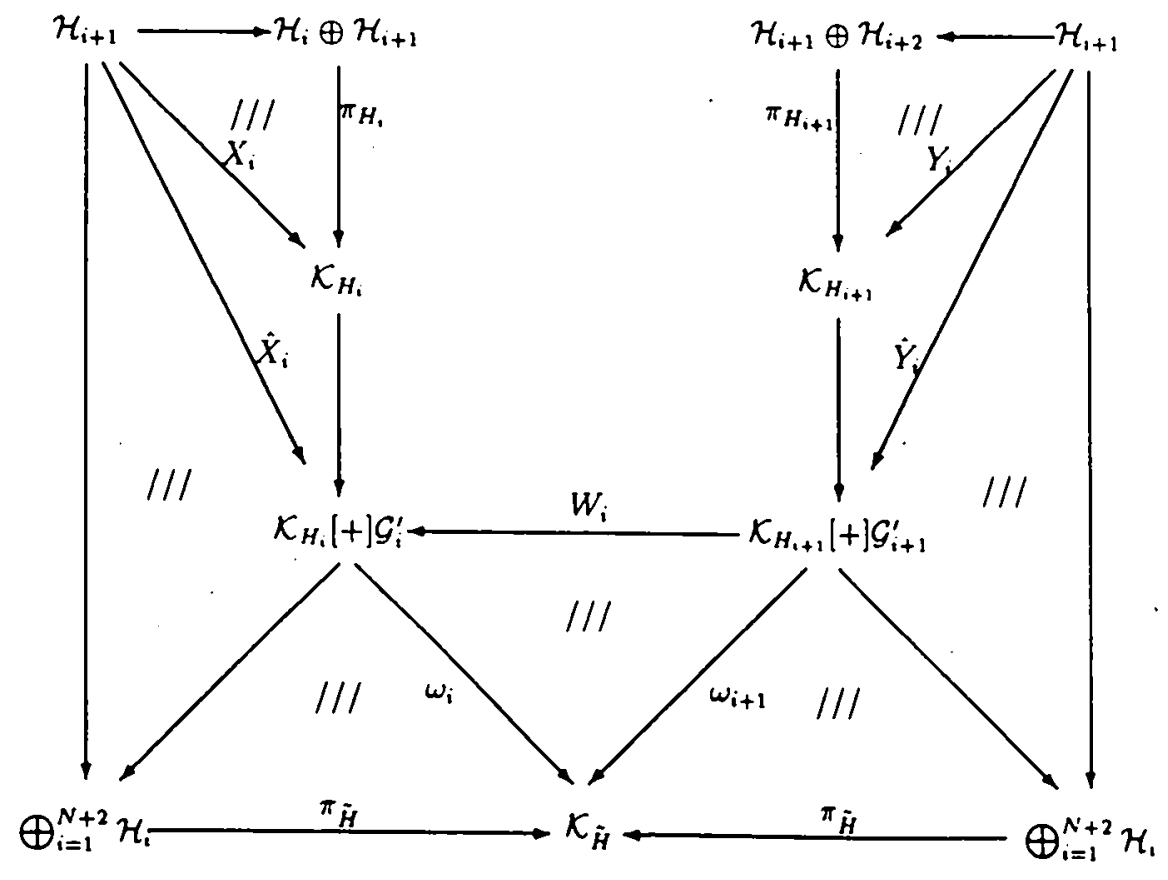

Figure 2 
Proposition 3.2. Let $\left(\tilde{H} ;\left(\mathcal{G}_{i}^{\prime}\right)_{i=1}^{N+1}\right)$ be a solution of the problem $\mathbf{C}(H ; \underline{\kappa})$ and let $\mathcal{X}$ and $\mathcal{Y}$ be defined as in (9.9). Then defining $W_{i}, X_{i}$ and $Y_{i}$ as in (9.10) and (9.11) the quadruple $(\underline{\mathcal{G}} ; \hat{\mathcal{X}}, \hat{\mathcal{Y}} ; \mathcal{W})$ is a solution of the problem $\operatorname{EF}(\mathcal{X}, \mathcal{Y} ; \underline{\kappa})$.

Proof. We use the diagram in Figure 2 in order to simplify the notation. We have to verify the requirements (a), (b), and (c) in the statement of the problem $\operatorname{EF}(\mathcal{X} ; \mathcal{Y} ; \underline{\kappa})$. The condition (a) is obvious. From (3.11) it is easy to verify that condition (b) holds. Using the same diagram we check immediately that condition (c)/(1) holds. In order to prove that the minimality condition (c)/(2) also holds we use the lower part of the corresponding diagrams for all $i \in\{1,2, \ldots, N\}$ and get

$$
\mathcal{K}_{H_{1}} \vee \bigvee_{i=1}^{N} W_{1} \cdots W_{i} \mathcal{K}_{H_{i+1}}=\omega^{-1} \pi_{\bar{H}} \mathcal{H}=\mathcal{K}_{H_{1}}|+| \mathcal{G}_{1}^{\prime}
$$

The proof is complete

In accordance with Proposition 3.2 and the results in Section 2 it is useful to introduce the orthogonal projections $P_{i}$ and $Q_{i}$ of $\mathcal{H}_{i+1}$ corresponding to those introduced in (2.4). In view of Proposition 3.2, $P_{i}$ is that onto the subspace $\overline{\mathcal{R}}\left(P_{\text {ker } A_{i+1, i+1}} A_{i, i+1}^{*}\right)$ and similarly $Q_{i}$ is that onto the subspace $\overline{\mathcal{R}}\left(P_{\text {ker } A_{i+1, i+1}} A_{i+1, i+2}^{*}\right)$.

Corollary 3.3. Assume that $\mathcal{K}_{A_{i+1, i+1}}$ is canonically embedded in both of $\mathcal{K}_{H_{i}}$ and $\mathcal{K}_{H_{i+1}}$, for all $i \in\{1,2, \ldots, N\}$, and assume also that all of the projections $P_{i} \wedge Q_{i}$ have finite ranks. Then, in order for the problem $\mathrm{C}(H ; \underline{\kappa})$ to be solvable it is necessary that the following conditions be satisfied:

$$
\begin{aligned}
\kappa_{i}+\operatorname{rank}\left(P_{i}\left(I-Q_{i}\right)\right)+\kappa^{-}\left[\mathcal{R}\left(\pi_{H_{i}} \mid \mathcal{H}_{i+1}^{\perp}\right]\right. \\
=\kappa_{i+1}+\operatorname{rank}\left(Q_{i}\left(I-P_{i}\right)\right)+\kappa^{-}\left[\mathcal{R}\left(\pi_{H_{i+1}} \mid \mathcal{H}_{i+1}^{\perp}\right]\right.
\end{aligned}
$$

$(i \in\{1,2, \ldots, N\})$ and

$$
\kappa_{i} \geq \max \left\{\operatorname{rank}\left(P_{i-1}\left(I-Q_{i-1}\right)\right), \operatorname{rank}\left(Q_{i}\left(I-P_{i}\right)\right)\right\}
$$

( $i \in\{1,2, \ldots, N+1\})$ with the notation $P_{0}=0$ and $Q_{N+1}=0$.

Proof. This is a direct consequence of Proposition 3.2 and Proposition 2.1

We have now to show that, in the framework established before, the solutions of the problem $\operatorname{EF}(\mathcal{X}, \mathcal{Y} ; \underline{\kappa})$ also produce solutions of the problem $\mathbf{C}(H ; \underline{\kappa})$.

Lemma 3.4. Let $\mathcal{X}$ and $\mathcal{Y}$ be defined as in (9.9) and also let $(\underline{\mathcal{G}} ; \hat{\mathcal{X}}, \hat{\mathcal{Y}} ; \mathcal{W})$ be a solution of the problem $\operatorname{EF}(\mathcal{X}, \mathcal{Y} ; \underline{\kappa})$. Consider the operator $\left.T_{1} \in \hat{\mathcal{L}}\left(\mathcal{H}, \mathcal{K}_{H_{1}} \mid+\right] \mathcal{G}_{1}^{\prime}\right)$ defined by

$$
T_{1} h= \begin{cases}\pi_{H_{1}} h & \text { for } h \in \mathcal{H}_{1} \\ W_{1} \cdots W_{k-2} \hat{X}_{k-1} h & \text { for } h \in \mathcal{H}_{k}(2 \leq k \leq N+1) \\ W_{1} \cdots W_{N \pi_{H_{+1}} h} & \text { for } h \in \mathcal{H}_{N+2}\end{cases}
$$

Then $T_{1}$ has dense range and $T_{1}^{\sharp} T_{1} \in \mathcal{L}(\mathcal{H})$ is a selfadjoint completion of $H$. 
Proof. The operator $T_{1}$ has dense range because $\mathcal{W}$ has the minimal property (c) $/(2)$. Clearly, $T_{1}^{\prime} T_{1}$ is selfadjoint on $\mathcal{H}$. We calculate now the entries of the matrix of $T_{1}^{d} T_{1}$ using (3.14). Firstly

$$
P_{\mathcal{H}_{1}} T_{1}^{\prime} T_{1}\left|\mathcal{H}_{1}=P_{\mathcal{H}_{1}} \pi_{H_{1}}^{l} \pi_{H_{1}}\right| \mathcal{H}_{1}=P_{\mathcal{H}_{1}} H_{1} \mid \mathcal{H}_{1}=A_{11}
$$

Taking into account that $\mathcal{R}\left(\hat{X}_{1}\right)$ is an extension of $\mathcal{R}\left(X_{1}\right)$ with a neutral subspace, we obtain

$$
P_{\mathcal{H}_{1}} T_{1}^{\S} T_{1}\left|\mathcal{H}_{2}=P_{\mathcal{H}_{1}} \pi_{H_{2}}^{\ell} \hat{X}_{1}=P_{\mathcal{H}_{1}} H_{1}\right| \mathcal{H}_{2}=A_{12}
$$

Using the property (b)/(2) we obtain

$$
P_{\mathcal{H}_{k}} T_{1}^{l} T_{1} \mid \mathcal{H}_{k}=\hat{X}_{k-1}^{!} \hat{X}_{k-1}=A_{k k} \quad(2 \leq k \leq N)
$$

Using the properties (b)/(2) and (b)/(3) of $\hat{X}_{k}$ and $\hat{Y}_{k}$ we obtain

$$
P_{\mathcal{H}_{k}} T_{1}^{t} T_{1} \mid \mathcal{H}_{k+1}=\hat{X}_{k-1}^{t} \dot{W}_{k-1} \hat{X}_{k}=\hat{Y}_{k-1}^{\prime} \hat{X}_{k}=A_{k, k+1} \quad(2 \leq k \leq N-1)
$$

Similarly we obtain

$$
P_{\mathcal{H}_{N+1}} T_{1}^{\prime} T_{1}\left|\mathcal{H}_{N+2}=\hat{X}_{N}^{\prime} \pi_{H_{N+1}}\right| \mathcal{H}_{N+2}=A_{N+1, N+2}
$$

and

$$
P_{\mathcal{H}_{N+2}} T_{1}^{l} T_{1}\left|\mathcal{H}_{N+2}=P_{\mathcal{H}_{N+2}} \pi_{\mathcal{H}_{N+1}}^{3} \pi_{H_{N+1}}\right| \mathcal{H}_{N+2}=A_{N+2, N+2}
$$

We thus proved that $T_{1}^{k} T_{1}$ is a selfadjoint completion of $H$

Proposition 3.5. Let $\mathcal{X}$ and $\mathcal{Y}$ be defined as in (S.1) and $\left(\hat{\mathcal{X}}, \hat{\mathcal{Y}} ; \mathcal{W}_{;}\left(\mathcal{G}_{i}^{\prime}\right)_{i=1}^{N+1}\right)$ be a solution of the problem $\operatorname{EF}(\mathcal{X}, \mathcal{Y} ; \underline{\kappa})$. If the operator $T_{1}$ is defined as in (s.14) and has the property $(\gamma)$, then $\left(T_{1}^{\prime} T_{1} ;\left(\mathcal{G}_{i}^{\prime}\right)_{i=1}^{N+1}\right)$ is a solution of the problem $\mathrm{C}(H ; \underline{\kappa})$.

Proof. Let us denote $\tilde{H} \stackrel{\prime}{=} T_{1}^{t} T_{1} \in \mathcal{L}(\mathcal{H})$. From Lemma 3.4 we know that $\tilde{H}$ is a selfadjoint completion of $H$. Since $T_{1}$ has dense range and taking into account that $T_{1}$ has the property $(\gamma)$ it follows that it uniquely yields a unitary operator $\omega_{1}$ : $\mathcal{K}_{H_{1}}(+] \mathcal{G}_{1}^{\prime} \rightarrow \mathcal{K}_{\dot{H}}$. From the definition of $T_{1}$ (see (3.14)) it follows that $\omega_{1}$ is an extension of the canonical embedding of $\mathcal{K}_{H_{1}}$ into $\mathcal{K}_{\bar{H}}$.

Further on, for $i \in\{1,2, \ldots, N+1\}$ we consider an operator $\dot{T}_{i} \in \mathcal{L}\left(\mathcal{H}, \mathcal{K}_{H_{1}}[+] \mathcal{G}_{i}^{\prime}\right)$ defined by .

$$
T_{i}=W_{i-1}^{\prime} \cdots W_{1}^{\prime} T_{1}
$$

Then we also have

$$
\tilde{H}=T_{i}^{\ell} T_{i}
$$

and all $T_{i}$ have the property $(\gamma)$. As before, we obtain from here a unitary operator $\omega_{i}: \mathcal{K}_{H_{i}}\left(+\mid \mathcal{G}_{i}^{\prime} \rightarrow \mathcal{K}_{\dot{H}}\right.$, which is an extension of the canonical embedding of $\mathcal{K}_{H_{i}}$ into $\mathcal{K}_{H_{H}}$. We proved thus that $\left(\tilde{H} ;\left(\mathcal{G}_{i}^{\prime}\right)_{i=1}^{N+1}\right)$ is a solution of the problem $\mathbf{C}(H ; \underline{\Lambda})$ 
We are now in a position to determine the minimal $\kappa$ for which the problem $\mathbf{C}(H ; \underline{\kappa})$ has solution. One of the main technical difficulties in applying Theorem 2.3 comes from the assumption in Proposition 3.5 that at least one factorization as in (3.16) produces unitary operators (i.e. at least one of $T_{i}$ has the property $(\gamma)$ ). This difficulty can be removed by restricting ourselves to the case

$$
\kappa^{-}(H)=\max _{i=1}^{N+1} \kappa^{-}\left(H_{i}\right) \leq \infty
$$

Let now $H$ be a selfadjoint tridiagonal partial block matrix operator such that (3.17) holds. We consider the families of operators $\mathcal{X}$ and $\mathcal{Y}$ defined as in (3.9) and notice that as a consequence of (3.17) all of the orthogonal projections $P_{i}$ and $Q_{i}$ onto

$$
\mathcal{R}\left(R_{\text {ker }\left(A_{i+1, i+1}\right)} H_{i}^{*} \mid \mathcal{H}_{i}\right) \quad \text { and } \quad \mathcal{R}\left(P_{\text {ker }\left(A_{i+1, i+1}\right)} H_{i+1}^{*} \mid \mathcal{H}_{i+1}\right),
$$

respectively, have finite ranks. Let us also consider the non-negative numbers $r_{i 1}$ and $r_{i 2}$ as in (2.12) and put $r_{01}=r_{N+1,2}=0$. In accordance with (2.13) we consider the numbers

$$
a_{i 1}=\kappa^{-}\left[\mathcal{R}\left(\pi_{H_{i}} \mid \mathcal{H}_{i+1}\right)^{\perp}\right] \quad \text { and } \quad a_{i 2}=\kappa^{-}\left[\mathcal{R}\left(\pi_{H_{i+1}} \mid \mathcal{H}_{i+1}\right)^{\perp}\right]
$$

and notice that their finiteness is a consequence of (3.17).

Theorem 3.6. Let $H=\left(H_{i}\right)_{i=1}^{N+1}$ be a selfadjoint tridiagonal partial block matrix operator such that (9.17) holds and let ws assume that $\mathcal{K}_{A_{i+1, i+1}}$ is canonically embedded in both of $\mathcal{K}_{H_{i}}$ and $\mathcal{K}_{H_{i+1}}$, for all $i \in\{1,2, \ldots, N\}$. Then, with the notation fixed before,

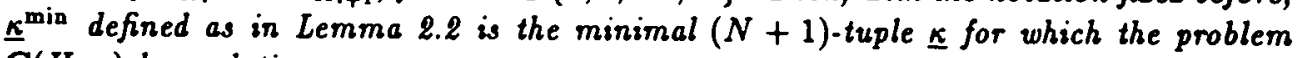
$\mathbf{C}(H ; \underline{\kappa})$ has solutions.

Proof. Indeed, using Proposition 3.2, the problem $\mathbf{C}(H ; \underline{\kappa})$ is reformulated as a problem of type $\operatorname{EF}(\mathcal{X}, \mathcal{Y} ; \underline{\kappa})$. The first part of Theorem 2.3 shows that for any $\underline{\kappa}$ such that problem $\mathbf{C}(H ; \underline{\kappa})$ has solutions we must have $\underline{\kappa} \geq \underline{\kappa}^{\min }$.

Conversely, recall that in Theorem 2.3 it is constructed a solution of the problem $\mathbf{E F}(\mathcal{X}, \mathcal{Y} ; \underline{\kappa})$. This produces a solution $\left(\tilde{H} ;\left(\mathcal{G}_{i}^{\prime}\right)_{i=1}^{N+1}\right)$ of the problem $\mathbf{C}\left(H ; \underline{\kappa}^{\mathrm{min}}\right)$ since we can apply Proposition 3.5, taking into account that $(3.17)$ yields the property $(\gamma)$ of $T_{1}$ (equivalently, all other $T_{i}$ have the property $(\gamma)$ ) due to a Pontryagin type Lemma argument (see, e.g., [16])

Remark. One can ask if the finiteness condition (3.17) is strong enough to insure the canonical embedding of $\mathcal{K}_{A_{i+1, i+1}}$ either into $\mathcal{K}_{H_{i+1}}$ or into $\mathcal{K}_{H_{i}}$. As it happens, this is not the case, due to the existence of unbounded isometric operators even in Pontryagin spaces (of different negative signatures), as some examples in [16] show. 


\section{The tridiagonal matrix case}

We consider now the case of matrices, i.e. all the operators $H_{i}$ and $A_{i j}$ are acting in finite-dimensional spaces (all Hilbert spaces $\mathcal{H}_{i}$ are finite-dimensional). Our aim will be, given a tridiagonal symmetric matrix $H$ as in (3.6), to calculate the number

$$
\min \left\{\kappa^{-}(\tilde{H}) \mid \tilde{H} \in \mathcal{C}_{h}(H)\right\}
$$

where $\mathcal{C}_{h}(H)$ denotes the set of all Hermitian completions $\tilde{H}$ of $H$. Thus we will apply the results obtained in the previous section but this time we will do this calculation in terms of

$$
\kappa^{-}(H)=\max _{i=1}^{N+1} \kappa^{-}\left(H_{i}\right)
$$

To this end let us first remark that we have

$$
\kappa^{-}\left(H_{i}\right)=\kappa^{-}\left[\mathcal{K}_{H_{i}}\right]
$$

where $\mathcal{K}_{H_{i}}$ denotes the (finite-dimensional) Kreln space induced by $H_{i}$. Also, we consider the orthogonal projections $\left\{P_{i}, Q_{i}\right\}$, where $P_{i}$ is the orthogonal projection onto the space $\mathcal{R}\left(R_{\operatorname{ker}\left(A_{i+1, i+1}\right)} H_{i}^{*} \mid \mathcal{H}_{i}\right)$ and, respectively, $Q_{i}$ is the orthogonal projection onto the space $\mathcal{R}\left(\mathcal{R}_{\operatorname{ler}\left(A_{i+1, i+1}\right)} H_{i+1}^{*} \mid \mathcal{H}_{i+1}\right)$. Using these we define the non-negative numbers $\left\{r_{i-1,2}, r_{i 1}\right\}_{i=1}^{N+1}$ by

$$
r_{i 1}=\operatorname{rank}\left(Q_{i}\left(I-P_{i}\right)\right) \quad \text { and } \quad r_{i 2}=\operatorname{rank}\left(P_{i}\left(I-Q_{i}\right)\right)
$$

and $r_{01}=r_{N+1,2}=0$. Let us also notice that in this finite-dimensional case the reduced Schur complements (see Lemma 3.1) exist and we can define

$$
a_{i 1}=\kappa^{-}\left(S_{r}\left(H_{i} ; A_{i, i+1}\right)\right) \quad \text { and } \quad a_{i 2}=\kappa^{-}\left(S_{r}\left(H_{i+1} ; A_{i, i+1}\right)\right)
$$

for all $i \in\{1,2, \ldots, N\}$.

We are now in a position to give a formula for computing the number defined in (4.1).

Theorem 4.1. Given a symmetric tridiagonal partial matrix $H$, consider the numbers defined in (4.4) and (4.5), and let $j \in\{1,2, \ldots, N\}$ be determined by the condition

$$
\begin{aligned}
\max & \left\{r_{j-1,2}, r_{j 1}\right\}+\sum_{k=1}^{j-1}\left[\left(a_{k 2}-a_{k 1}\right)+\left(r_{k 1}-r_{k 2}\right)\right] \\
& =\max _{n=1}^{N+1}\left\{\max \left\{r_{n-1,2}, r_{n 1}\right\}+\sum_{k=1}^{n-1}\left[\left(a_{k 2}-a_{k 1}\right)+\left(r_{k 1}-r_{k 2}\right)\right]\right\} .
\end{aligned}
$$

Then

$$
\min \left\{\kappa^{-}(\tilde{H}) \mid \tilde{H} \in \mathcal{C}_{h}(H)\right\}=\kappa^{-}\left(H_{j}\right)+\max \left\{r_{j-1,2}, r_{j 1}\right\}
$$

Proof. Let us first notice that since all the spaces occuring here are finite-dimensional, the conditions of canonical embeddings of different induced Kreln spaces are automatically satisfied. On the other hand, it is easy to see that $\tilde{H}$ belongs to $C_{A}(B)$ if and only if there exist $\left(\mathcal{G}_{i}^{\prime}\right)_{i=1}^{N+1}$ such that $\left(\tilde{H} ; \mathcal{G}_{i}^{\prime}\right)_{i=1}^{N+1}$ is a solution of the problem $\mathrm{C}(H ; \underline{\kappa})$, where $\underline{\kappa}=\left(\kappa_{i}\right)_{i=1}^{N+1}$ is defined by

$$
\kappa_{i}=\kappa^{-}(\tilde{H})-\kappa^{-}\left(H_{i}\right) \quad(i \in\{1,2, \ldots, N+1\}) .
$$

This observation enables us to apply Theorem 3.6 and obtain (4.7), where the index $j$ is determined by the condition (4.6) 
Remark 4.2. With the notation in Theorem 4.1, let the $(N+1)$-tuple $\underline{\kappa}^{\text {min }}$ be defined as in (2.11). Then

$$
\min \left\{\kappa^{-}(\tilde{H}) \mid \tilde{H} \in \mathcal{C}_{h}(H)\right\}=\kappa^{-}\left(H_{k}\right)+\kappa_{k}^{\min }
$$

for any other $k \in\{1,2, \ldots, N+1\}$.

Remark 4.3. Letting $\underline{\kappa}^{\mathrm{min}}$ be defined as in Lemma 2.2, from Theorem 4.1 and the previous remark it follows

$$
\min \left\{\kappa^{-}(\tilde{H}) \mid \tilde{H} \in \mathcal{C}_{h}(H)\right\}=\kappa^{-}(H)+\min _{i=1}^{N+1} \kappa_{i}^{\min }
$$

Also let us notice that the index $p$ which realizes the maximum in

$$
\kappa^{-}(H)=\max _{k=1}^{N+1} \kappa^{-}\left(H_{k}\right)
$$

is the same as the index $p$ realizing $\min \left\{\kappa_{i}^{\min } \mid i \in\{1 ; 2, \ldots, N+1\}\right\}$ and this index $p$ can be also determined by the condition.

$$
\sum_{k=1}^{p-1}\left[\left(a_{k 2}-a_{k 1}\right)+\left(r_{k 1}-r_{k 2}\right)\right]=\max _{l=1}^{N+1}\left\{\sum_{k=1}^{l-1}\left[\left(a_{k 2}-a_{k 1}\right)+\left(r_{k 1}-r_{k 2}\right)\right]\right\} .
$$

This also shows that the index $p$ is in general different of the index $j$ which realizes the maximum in (4.6).

We are now interested in finding a condition assuring that the minimal negative signature is preserved by completion. This is possible by applying the results obtained up to now.

Corollary 4.4. With the notation in Theorem 4.1, there exists $\tilde{H} \in \mathcal{C}_{h}(H)$ such that $\kappa^{-}(\tilde{H})=\kappa^{-}(H)$ if and only if for $j$ determined by the condition (4.5) and $p$ determined by the condition (4.11), we have either

$$
\max \left\{r_{j-1,2}, r_{j} 1\right\}+\sum_{k=p}^{j-1}\left[\left(a_{k 2}-a_{k 1}\right)+\left(r_{k 1}-r_{k 2}\right)\right]=0
$$

if $p \leq j$, or

$$
\max \left\{r_{j-1,2}, r_{j} 1\right\}+\sum_{k=j}^{p-1}\left[\left(a_{k 2}-a_{k 1}\right)+\left(r_{k 1}-r_{k 2}\right)\right]=0
$$

if $p \geq j$.

Proof. We use the previous remark and Theorem 4.1

Once the number in (4.1) is determined we can ask for determining a completion $\tilde{H} \in \mathcal{C}_{h}(H)$ which realizes the minimum of the negative squares. Tracing back to Theorem 2.3 we notice that such a completion can be obtained by means of the formula (3.17) and the construction in the proof of Theorem 2.3. 


\section{References}

[1] Arsene, Gr., Constantinescu, T. and A. Gheondea: Lifting of operators and prescribed numbers of negative squares. Michigan Math. J. 34 (1987), 201 - 216.

[2] Azizov, T. Ya. and I. S. Iokhvidov: Foundations of the Theory of Linear Operators in Spaces with Indefinite Metrics (in Russian). Moscow: Nauka 1986.

[3] Bognár, J.: Indefinite Inner Product Spaces. Berlin - Heidelberg - New York: SpringerVerlag 1974.

[4] Constantinescu, T.: Completions and extensions. Oper. Theory: Adv. Applic. 43 (1990), $141 \cdot 150$.

[5] Constantinescu, T. and A. Gheondea: The negative signature of some Hermitian matrices. Lin. Algebra Appl. 178 (1993), 17 - 42.

[6] Constantinescu, T. and A. Gheondea: Elementary rotations of linear operators in Krein spaces. J. Oper. Theory (to appear).

[7] Constantinescu, T. and A. Gheondea: Extending factorizations and minimal negative signatures. J. Oper. Theory (to appear).

[8] Dancis, J.: The possible inertia for a Hermitian matrix and its principal submatrices. Lin. Alg. Appl. 85 (1987), 121 - 151.

[9] Dancis, J.: Bordered matrices. Lin. Alg. Appl. 128 (1990), 117 - 132.

[10] Dym, H. and I. Gohberg: Extensions of band matrices with band inverses. Lin. Algebra Appl. 36 (1981), 1 - 24.

[11] Ellis, R., Gohberg, I. and D. C. Lay: On negative eigenvalues of selfadjoint extensions of band matrices. Lin. Multilin. Alg. 24 (1988), $15-25$.

[12] Gheondea, A.: One-step completions of Hermitian partial matrices with minimal negative signature. Lin. Alg. Appl. 173 (1992), 99 - 114.

[13] Gohberg, I., Kaashoek, M. A. and H. J. Woerdeman: A Note on extensions of band matrices with maximal and submaximal invertible blocks. Lin. Alg. Appl. 150 (1991), 157 - 166.

[14] Golumbic, M. C.: Algorithmic Graph Theory and Perfect Graphs. New York: Academic Press 1980.

[15] Grone, R., Johnson, C. R., de Sá, E. M. and H. Wolkowiecz: Positive definite completions of partial Hermitian matrices. Lin. Alg. Appl. 58 (1984), 109 - 124.

[16] Iokhvidov, I. S., Krěn, M. G. and H. Langer: Introduction to the Spectral Theory of Operators in Spaces with Indefinite Metric. Berlin: Akademie-Verlag 1982.

[17] Johnson, C. R. and L. Rodman: Inertia possibilities for completions of partial Hermitian matrices. Lin. Multilin. Alg. 16 (1984), 179 - 195.

[18] Maddocks, J.: Restricted quadratic forms, inertia theorems, and Schur complements. Lin. Alg. Appl. 108 (1988), 1 - 36.

Received 01.07.1993 